\title{
Estimating the Ultimate Bearing Capacity for Strip Footing Near and within Slopes Using AI (GP, ANN, and EPR) Techniques
}

\author{
Ahmed M. Ebid $\mathbb{D},{ }^{1}$ Kennedy C. Onyelowe $\mathbb{D}^{2},{ }^{2}$ and Emmanuel E. Arinze ${ }^{3}$ \\ ${ }^{1}$ Department of Structural Engineering, Faculty of Engineering and Technology, Future University, New Cairo, Egypt \\ ${ }^{2}$ Department of Civil and Mechanical Engineering, Kampala International University, Kampala, Uganda \\ ${ }^{3}$ Department of Civil Engineering, Michael Okpara University of Agriculture, Umudike, Nigeria \\ Correspondence should be addressed to Kennedy C. Onyelowe; kennedychibuzor@kiu.ac.ug
}

Received 17 July 2021; Accepted 26 October 2021; Published 9 November 2021

Academic Editor: Xing Ma

Copyright (c) 2021 Ahmed M. Ebid et al. This is an open access article distributed under the Creative Commons Attribution License, which permits unrestricted use, distribution, and reproduction in any medium, provided the original work is properly cited.

\begin{abstract}
Numerical and computational analyses surrounding the behavior of the bearing capacity of soils near or adjacent to slopes have been of great importance in earthwork constructions around the globe due to its unique nature. This phenomenon is encountered on pavement vertical curves, drainages, and vertical infrastructure foundations. In this work, multiple data were collected on the soil and footing interface parameters, which included width of footing, depth of foundation, distance of slope from the footing edge, soil bulk density, slope and frictional angles, and bearing capacity factors of cohesion and overburden pressure determined for the case of a foundation on or adjacent to a slope. The genetic programming (GP), evolutionary polynomial regression (EPR), and artificial neural network (ANN) intelligent techniques were employed to predict the ultimate bearing capacity of footing on or adjacent to a slope. The performance of the models was evaluated as well as compared their accuracy and robustness with the findings of Prandtl. The results were observed to show the superiority of GP, EPR, and ANN techniques over the computational works of Prandtl. In addition, the ANN outclassed the other artificial intelligence methods in the exercise.
\end{abstract}

\section{Introduction}

Building substructures are often constructed on or adjacent to slopes owing to the nonavailability of level ground, especially in hilly dominant topography encountered in highway vertical curves, embankments, erosion watersheds, etc. The study of the bearing capacity of loaded slopes is vital because they are more prone to fail than other types of earth structures [1-5]. Shallow foundations on slopes are mainly used for small- to medium-rise buildings. In frictional soils, the bearing capacity is mainly governed by foundation failure, while in cohesive soils, the bearing capacity of the foundation is controlled by the stability of the soil structure [6-9]. Recently, methods proposed by the researchers available to find the bearing capacity of shallow foundations on or near slopes include limit equilibrium analysis $[10,11]$, slip line analysis [12], variational calculus [13], the method of rigorous characteristics [14], improved movement optimization [15], finite element analysis [16, 17], and multiblock analysis [9]. Determining the bearing capacity of a shallow substructure is a very important component of geotechnical engineering study and practice. Prandtl [18] is generally credited with some pioneer work in bearing capacity theory, having tried to establish the punching failure mechanism for thick metals based on the theory of plasticity. This theory gave rise to Terzaghi's [19] work, where the effects of soil internal angle of friction, soil cohesion, and surcharge (overburden pressure) were taken into consideration, giving rise to the bearing capacity factors $(N c, N \gamma$, and $N q$ ). Meyerhof [20] improved on Terzaghi's superimposed theory to account for soil strength, footing size and shape, and slope. Vesic later considered the effects of shallow foundation shape on the ultimate bearing capacity [21]. Nevertheless, despite studies into the effects of foundation shape and depth [19], Meyerhof (1957 and 1974) [20-23], there has been few research works on the bearing capacity of 
footings on and/or adjacent to slopes made of $c^{\prime}-\mathrm{p}^{\prime}$ soils. Meyerhof studied general failure mechanisms for bearing capacity on purely cohesionless or cohesive soils adjacent to slopes using an assumed failure pattern based on the empirical observation from model footing tests in the laboratory. However, actual quantifiable results were not available for model verifications. Graham et al. [24] derived an analytical model for bearing capacity and stress distribution for footings placed upon slopes for cohesionless soils that was validated by experimental results. However, his work did not expand to soils with both cohesion and internal friction. Raj and Bharathi reported that Bowles provided a set of revised bearing capacity factors for $c^{\prime}-\phi^{\prime}$ soils based on an the assumed failure mechanism resembling that from Terzaghi, but limited to set of strength values. Griffiths [25] used the finite element analysis (FEA) to determine the bearing capacity of $c^{\prime}-\phi^{\prime}$ soils on slopes attaining considerable results. However, his $N \gamma$ factor recorded pitfall due to a convergence issue. Applying upper bound limits analysis, Kusakabe et al. [26] presented a series of design charts with dimensionless factors showing a reduction in bearing capacity compared to the conventional bearing capacity approach for footings placed on the level of the ground for soils with and without cohesion. A better analysis of the complex, complicated failure mechanism associated with the ultimate bearing capacity of strip footings on slopes requires use of tools that can account for the complex failure kinematics related to the soil-structure interaction and nonintuitive behavior of the soil collapse mechanism [27, 28]; in this case, artificial intelligence (AI) looks a better tool.

Recent discoveries in the field of artificial intelligence (AI) applications have risen in the development of accurate and dependable models for solving engineering problems [29-32]. Advances in the field of AI made it possible to produce models to adapt to difficulties associated with modelling soil and foundation behavior [33-36]. AI has been considered in the field of civil engineering for more than one and a half decade [37]. These models involve a simple black-box model to complex distributed physicsbased models. Although there are numerous AI modelling algorithms, namely, genetic algorithm (GA), ant colony (AC), differential evolution (DE), particle swarm (PS), artificial neural network (ANN), genetic programming (GP) [35], and gene expression programming [32], artificial neural network (ANN) [36], genetic programming (GP), and gene expression programming have been widely used $[29,35]$.

\section{Methodology}

2.1. Preamble, Data Collection, and Statistical Study. Prantdl [18] proposed a mathematical formula for the ultimate bearing capacity of strip footing in a semi-infinity soil model with a horizontal ground surface based on an experimentally observed failure shape below footing as shown in equation (1). For strip footing near or within the slope, Prantdl suggested modified parameters $N^{\prime} c=N c$ $(L 1 / L 0)$ and $N^{\prime} q=N q(A 1 / A 0)$, where $L 0, L 1, A 0$, and $A 1$ are illustrated in Figure 1 and could be calculated from the geometry. $N \gamma$ is still the same because the triangular wedges below the footings are the same in all cases. The aim of this research is to develop mathematical formulas for the modified $N^{\prime} c$ and $N^{\prime} q$ based on slope geometry and soil proprieties using different artificial intelligence (AI) techniques.

$$
q_{\mathrm{ult}}=C N c+q N q+\gamma B N \gamma
$$

where qult: ultimate bearing capacity of strip footing, $C$ : soil cohesion, $q$ : overburden pressure at foundation depth, $\gamma$ : bulk density of soil below the footing, B: strip footing width, $N c, N q$, and $N \gamma$ are parameters based on the soil internal friction angle $\phi, N q: e \pi \tan \phi \cdot \tan 2(45+\phi / 2), N c:(N q-1)$ $\cot \phi(N c=5$ for $\phi=0)$, and $N \gamma:(N q-1) \tan \phi$.

From the foregoing, the methodology was to generate a database of 300 records; each record contains slope angle $(\beta)$, angle of internal friction of soil $(\phi)$, ratio between the distance to the slope edge and footing width $(b / B)$, and the ratio between foundation depth and footing width $(D f / B)$ besides the calculated values for both $N^{\prime} c$ and $N^{\prime} q$. Table 1 includes the complete dataset, while Tables 2 and 3 summarize their statistical characteristics and the Pearson correlation matrix. Finally, Figure 2 shows the histograms for both inputs and outputs.

2.2. Research Program. Three different artificial intelligent (AI) techniques were used to predict the values of both $N^{\prime} c$ and $N^{\prime} q$. These techniques are genetic programming (GP), artificial neural network (ANN), and polynomial linear regression optimized using genetic algorithm which is known as evolutionary polynomial regression (EPR). All the three developed models were based on the generated database Each model of the three developed models was based on different approaches (evolutionary approach for GP, mimicking biological neurons for ANN, and an optimized mathematical regression technique for EPR). However, for all developed models, prediction accuracy was evaluated in terms of sum of squared errors (SSE).

The following section discusses the results of each model. The accuracies of the developed models were evaluated by comparing the SSE between the predicted and calculated $N^{\prime} c$ and $N^{\prime} q$ values.

\section{Predictive Model Results}

\subsection{Prediction of $N^{\prime} c$ and $N^{\prime} q$}

3.1.1. Model (1)-Using the GP Technique. The developed GP model started with the one level of complexity and settled at five levels of complexity. The population size, survivor size, and number of generations were 10000, 30000, 50000, 75000, and 100000, respectively. Equations (2) and (3) present the output formulas for $N^{\prime} c$ and $N^{\prime} q$, while Figures 3(a) and 4(a) show their fitness. The average errors (\%) of the total set are $20.7 \%$ and $31.6 \%$, respectively, while the corresponding $\left(R^{2}\right)$ values are 0.951 and 0.949 . 


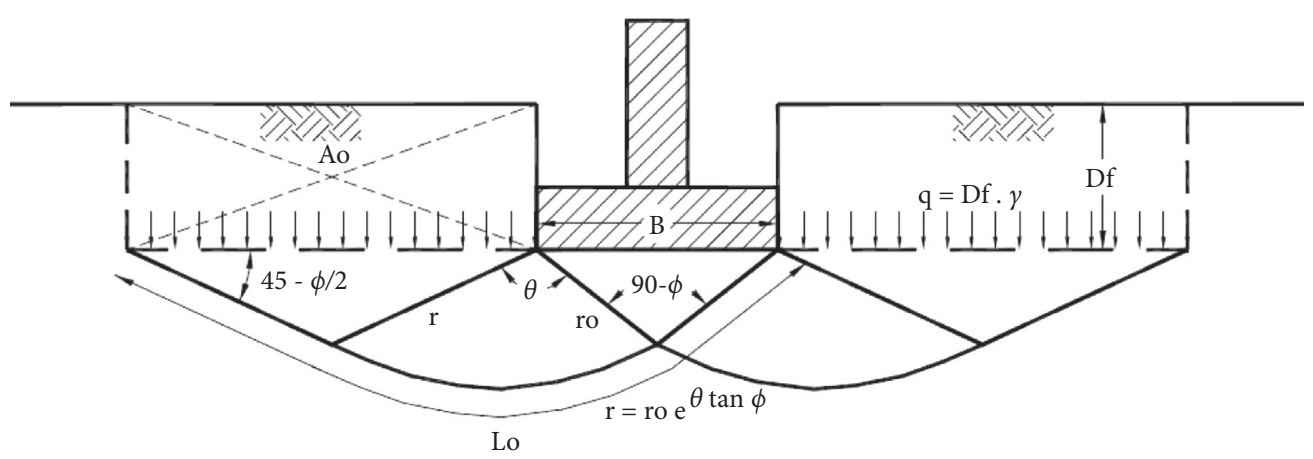

(a)

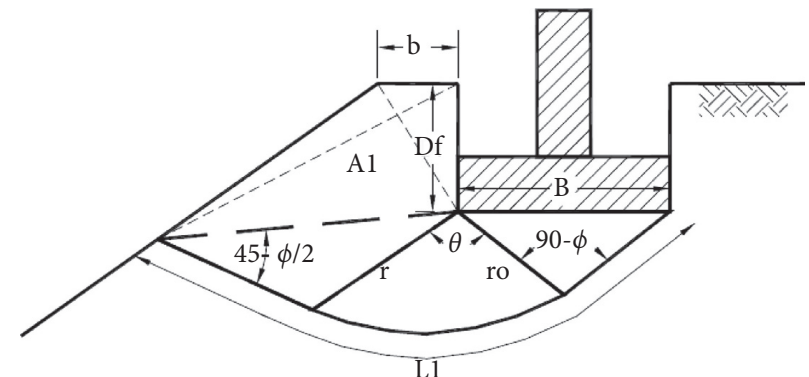

(b)

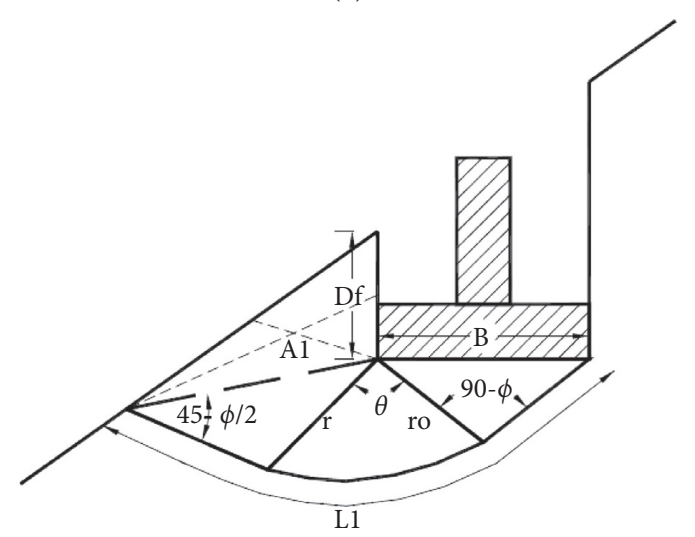

(c)

Figure 1: Shear failure mechanism for soil below strip footing after Prantdl [18]. (a) Case of strip footing with a horizontal ground surface. (b) Case of strip footing near the slope. (c) Case of strip footing within the slope.

$$
\begin{aligned}
& N^{\prime} c=e^{(5+\phi) / 11}+\left(7+\frac{b}{B}+\frac{D_{f}}{B}\right)-\left(\frac{7+\beta}{7+D_{f} / B}\right) \\
& N^{\prime} q=e^{\left(\phi-11 / 7+D_{f} / B\right)}-\left(\frac{\beta}{11\left(3-D_{f} / B\right)}\right)+\left(\left(3^{b / B}\right)\left(\frac{D_{f}}{B^{\left(D_{f} / B\right)}}\right)\right) .
\end{aligned}
$$

3.1.2. Model (2)-Using the ANN Technique. A back-propagation ANN with one hidden layer and Hyper Tan activation function was used to predict the values of N'c and N'q. The used network layout is illustrated in Figure 5, and its connation weights are listed in Table 4 . Since the used ANN has a nonlinear activation function, the equivalent equation is very complicated to be presented mathematically. The average errors in \% of this network are $6.9 \%$ and $13.0 \%$ for $N^{\prime} c$ and
$N^{\prime} q$, respectively, while the corresponding $R^{2}$ values are 0.995 and 0.991 . The relation between the calculated and predicted values is shown in Figures 3(b) and 4(b). The summation of connection weights of each input parameter is a good indication for its importance; accordingly, it was found that $\phi$ was the most important parameter with $78.6 \%$ of the total weights. $\beta$ came in the second place with $10.1 \%$ and then $b / B$ and $D_{f} / B$ with $7.7 \%$ and $3.6 \%$, respectively. 
TABLE 1: The used database.

\begin{tabular}{|c|c|c|c|c|c|}
\hline$(b / B)$ & $\left(D_{f} / B\right)$ & $\beta$ & $\phi$ & $N^{\prime} c$ & $N^{\prime} q$ \\
\hline 0.0 & 0.0 & 0.0 & 0.0 & 5.14 & 1.03 \\
\hline 0.0 & 0.0 & 0.0 & 10.0 & 8.34 & 2.47 \\
\hline 0.0 & 0.0 & 0.0 & 20.0 & 14.83 & 6.40 \\
\hline 0.0 & 0.0 & 0.0 & 30.0 & 30.14 & 18.40 \\
\hline 0.0 & 0.0 & 0.0 & 40.0 & 75.31 & 64.20 \\
\hline 0.0 & 0.0 & 5.0 & 0.0 & 5.01 & 1.03 \\
\hline 0.0 & 0.0 & 5.0 & 10.0 & 8.07 & 2.47 \\
\hline 0.0 & 0.0 & 5.0 & 20.0 & 14.19 & 6.40 \\
\hline 0.0 & 0.0 & 5.0 & 30.0 & 28.43 & 18.40 \\
\hline 0.0 & 0.0 & 5.0 & 40.0 & 69.67 & 64.20 \\
\hline 0.0 & 0.0 & 10.0 & 0.0 & 4.89 & 1.03 \\
\hline 0.0 & 0.0 & 10.0 & 10.0 & 7.80 & 2.47 \\
\hline 0.0 & 0.0 & 10.0 & 20.0 & 13.57 & 6.40 \\
\hline 0.0 & 0.0 & 10.0 & 30.0 & 26.80 & 18.40 \\
\hline 0.0 & 0.0 & 10.0 & 40.0 & 64.42 & 64.20 \\
\hline 0.0 & 0.0 & 20.0 & 0.0 & 4.63 & 1.03 \\
\hline 0.0 & 0.0 & 20.0 & 10.0 & 7.28 & 2.47 \\
\hline 0.0 & 0.0 & 20.0 & 20.0 & 12.39 & 6.40 \\
\hline 0.0 & 0.0 & 20.0 & 30.0 & 23.78 & 18.40 \\
\hline 0.0 & 0.0 & 20.0 & 40.0 & 55.01 & 64.20 \\
\hline 0.0 & 0.0 & 30.0 & 0.0 & 4.38 & 1.03 \\
\hline 0.0 & 0.0 & 30.0 & 10.0 & 6.77 & 2.47 \\
\hline 0.0 & 0.0 & 30.0 & 20.0 & 11.28 & 6.40 \\
\hline 0.0 & 0.0 & 30.0 & 30.0 & 21.05 & 18.40 \\
\hline 0.0 & 0.0 & 30.0 & 40.0 & 55.01 & 64.20 \\
\hline 0.0 & 0.0 & 60.0 & 0.0 & 3.62 & 1.03 \\
\hline 0.0 & 0.0 & 60.0 & 10.0 & 5.33 & 2.47 \\
\hline 0.0 & 0.0 & 60.0 & 20.0 & 8.33 & 6.40 \\
\hline 0.0 & 0.0 & 60.0 & 30.0 & 14.34 & 18.40 \\
\hline 0.0 & 0.0 & 60.0 & 40.0 & 28.56 & 64.20 \\
\hline 0.5 & 0.5 & 5.0 & 0.0 & 5.14 & 0.83 \\
\hline 0.5 & 0.5 & 5.0 & 10.0 & 8.34 & 1.85 \\
\hline 0.5 & 0.5 & 5.0 & 20.0 & 14.83 & 4.41 \\
\hline 0.5 & 0.5 & 5.0 & 30.0 & 30.14 & 11.67 \\
\hline 0.5 & 0.5 & 5.0 & 40.0 & 75.31 & 37.38 \\
\hline 0.5 & 0.5 & 10.0 & 0.0 & 5.14 & 0.94 \\
\hline 0.5 & 0.5 & 10.0 & 10.0 & 8.34 & 2.03 \\
\hline 0.5 & 0.5 & 10.0 & 20.0 & 14.93 & 4.73 \\
\hline 0.5 & 0.5 & 10.0 & 30.0 & 30.14 & 12.17 \\
\hline 0.5 & 0.5 & 10.0 & 40.0 & 74.12 & 37.80 \\
\hline 0.5 & 0.5 & 20.0 & 0.0 & 5.14 & 1.03 \\
\hline 0.5 & 0.5 & 20.0 & 10.0 & 8.34 & 2.34 \\
\hline 0.5 & 0.5 & 20.0 & 20.0 & 14.83 & 5.19 \\
\hline 0.5 & 0.5 & 20.0 & 30.0 & 29.79 & 12.66 \\
\hline 0.5 & 0.5 & 20.0 & 40.0 & 65.74 & 36.91 \\
\hline 0.5 & 0.5 & 30.0 & 0.0 & 5.14 & 1.03 \\
\hline 0.5 & 0.5 & 30.0 & 10.0 & 8.34 & 2.47 \\
\hline 0.5 & 0.5 & 30.0 & 20.0 & 14.83 & 5.36 \\
\hline 0.5 & 0.5 & 30.0 & 30.0 & 27.64 & 12.40 \\
\hline 0.5 & 0.5 & 30.0 & 40.0 & 58.32 & 34.02 \\
\hline 0.5 & 0.5 & 60.0 & 0.0 & 5.14 & 1.03 \\
\hline 0.5 & 0.5 & 60.0 & 10.0 & 8.07 & 2.13 \\
\hline 0.5 & 0.5 & 60.0 & 20.0 & 12.37 & 3.92 \\
\hline 0.5 & 0.5 & 60.0 & 30.0 & 20.74 & 7.83 \\
\hline 0.5 & 0.0 & 5.0 & 0.0 & 5.14 & 1.03 \\
\hline 0.5 & 0.0 & 5.0 & 10.0 & 8.25 & 2.47 \\
\hline 0.5 & 0.0 & 5.0 & 20.0 & 14.45 & 6.40 \\
\hline 0.5 & 0.0 & 5.0 & 40.0 & 70.40 & 64.20 \\
\hline 0.5 & 0.0 & 10.0 & 0.0 & 5.14 & 1.03 \\
\hline 0.5 & 0.0 & 10.0 & 10.0 & 8.15 & 2.47 \\
\hline
\end{tabular}

TABle 1: Continued.

\begin{tabular}{lccccc}
\hline$(b / B)$ & $\left(D_{f} / B\right)$ & $\beta$ & $\phi$ & $N^{\prime} c$ & $N^{\prime} q$ \\
\hline 0.5 & 0.0 & 10.0 & 20.0 & 14.08 & 6.40 \\
0.5 & 0.0 & 10.0 & 30.0 & 27.62 & 18.40 \\
0.5 & 0.0 & 10.0 & 40.0 & 65.87 & 64.20 \\
0.5 & 0.0 & 20.0 & 0.0 & 5.12 & 1.03
\end{tabular}

$\begin{array}{llllll}0.5 & 0.0 & 20.0 & 10.0 & 6.96 & 2.47\end{array}$

$\begin{array}{llllll}0.5 & 0.0 & 20.0 & 20.0 & 13.40 & 6.40\end{array}$

$\begin{array}{llllll}0.5 & 0.0 & 20.0 & 30.0 & 25.39 & 18.40\end{array}$

$\begin{array}{llllll}0.5 & 0.0 & 20.0 & 40.0 & 57.86 & 64.20\end{array}$

$\begin{array}{llllll}0.5 & 0.0 & 30.0 & 0.0 & 5.10 & 1.03\end{array}$

$\begin{array}{llllll}0.5 & 0.0 & 30.0 & 10.0 & 7.77 & 2.47\end{array}$

$\begin{array}{llllll}0.5 & 0.0 & 30.0 & 20.0 & 12.75 & 6.40\end{array}$

$\begin{array}{llllll}0.5 & 0.0 & 30.0 & 30.0 & 23.40 & 18.40\end{array}$

$\begin{array}{llllll}0.5 & 0.0 & 30.0 & 40.0 & 51.07 & 64.20\end{array}$

$\begin{array}{llllll}0.5 & 0.0 & 60.0 & 0.0 & 4.86 & 1.03\end{array}$

$\begin{array}{llllll}0.5 & 0.0 & 60.0 & 10.0 & 7.07 & 2.47\end{array}$

$\begin{array}{llllll}0.5 & 0.0 & 60.0 & 20.0 & 10.89 & 6.40\end{array}$

$\begin{array}{llllll}0.5 & 0.0 & 60.0 & 30.0 & 18.40 & 18.40\end{array}$

$\begin{array}{llllll}0.5 & 0.0 & 60.0 & 40.0 & 35.81 & 64.20\end{array}$

$\begin{array}{llllll}1.0 & 0.5 & 5.0 & 0.0 & 5.14 & 0.86\end{array}$

$\begin{array}{llllll}1.0 & 0.5 & 5.0 & 10.0 & 8.34 & 1.95\end{array}$

$\begin{array}{llllll}1.0 & 0.5 & 5.0 & 20.0 & 14.83 & 4.70\end{array}$

$\begin{array}{llllll}1.0 & 0.5 & 5.0 & 30.0 & 30.14 & 12.53\end{array}$

$\begin{array}{llllll}1.0 & 0.5 & 5.0 & 40.0 & 75.31 & 40.33\end{array}$

$\begin{array}{llllll}1.0 & 0.5 & 10.0 & 0.0 & 5.14 & 1.01\end{array}$

$\begin{array}{llllll}1.0 & 0.5 & 10.0 & 10.0 & 8.34 & 2.26\end{array}$

$\begin{array}{llllll}1.0 & 0.5 & 10.0 & 20.0 & 14.83 & 5.35\end{array}$

$\begin{array}{llllll}1.0 & 0.5 & 10.0 & 30.0 & 30.14 & 13.94\end{array}$

$\begin{array}{llllll}1.0 & 0.5 & 10.0 & 40.0 & 75.31 & 43.57\end{array}$

$\begin{array}{llllll}1.0 & 0.5 & 20.0 & 0.0 & 5.14 & 1.03\end{array}$

$\begin{array}{llllll}1.0 & 0.5 & 20.0 & 10.0 & 8.34 & 2.47\end{array}$

$\begin{array}{llllll}1.0 & 0.5 & 20.0 & 20.0 & 14.83 & 6.40\end{array}$

$\begin{array}{llllll}1.0 & 0.5 & 20.0 & 30.0 & 30.14 & 16.29\end{array}$

$\begin{array}{llllll}1.0 & 0.5 & 20.0 & 40.0 & 68.61 & 47.76\end{array}$

$\begin{array}{llllll}1.0 & 0.5 & 30.0 & 0.0 & 5.14 & 1.03\end{array}$

$\begin{array}{llllll}1.0 & 0.5 & 30.0 & 10.0 & 8.34 & 2.47\end{array}$

$\begin{array}{llllll}1.0 & 0.5 & 30.0 & 20.0 & 14.83 & 6.40\end{array}$

$\begin{array}{llllll}1.0 & 0.5 & 30.0 & 30.0 & 29.80 & 17.81\end{array}$

$\begin{array}{llllll}1.0 & 0.5 & 30.0 & 40.0 & 62.51 & 48.90\end{array}$

$\begin{array}{llllll}1.0 & 0.5 & 60.0 & 0.0 & 5.14 & 1.03\end{array}$

$\begin{array}{llllll}1.0 & 0.5 & 60.0 & 10.0 & 8.34 & 2.47\end{array}$

$\begin{array}{llllll}1.0 & 0.5 & 60.0 & 20.0 & 14.83 & 6.40\end{array}$

$\begin{array}{llllll}1.0 & 0.5 & 60.0 & 30.0 & 24.80 & 15.66\end{array}$

$\begin{array}{llllll}1.0 & 0.5 & 60.0 & 30.0 & 24.80 & 15.66 \\ 1.0 & 0.5 & 60.0 & 40.0 & 47.25 & 35.82\end{array}$

$\begin{array}{llllll}1.0 & 0.0 & 5.0 & 0.0 & 5.14 & 1.03\end{array}$

$\begin{array}{llllll}1.0 & 0.0 & 5.0 & 10.0 & 8.34 & 2.47\end{array}$

$\begin{array}{llllll}1.0 & 0.0 & 5.0 & 10.0 & 8.34 & 2.47 \\ 1.0 & 0.0 & 5.0 & 20.0 & 14.71 & 6.40\end{array}$

$\begin{array}{llllll}1.0 & 0.0 & 5.0 & 30.0 & 29.25 & 18.40\end{array}$

$\begin{array}{llllll}1.0 & 0.0 & 5.0 & 40.0 & 71.13 & 64.20\end{array}$

$\begin{array}{cccccc}1.0 & 0.0 & 10.0 & 0.0 & 5.14 & 1.03\end{array}$

$\begin{array}{llllll}1.0 & 0.0 & 10.0 & 10.0 & 8.34 & 2.47\end{array}$

$\begin{array}{cccccc}1.0 & 0.0 & 10.0 & 20.0 & 14.60 & 6.40 \\ 1.0 & 0.0 & 10.0 & 30.0 & 29.43 & 18.40\end{array}$

$\begin{array}{llllll}1.0 & 0.0 & 10.0 & 40.0 & 67.33 & 64.20\end{array}$

$\begin{array}{llllll}1.0 & 0.0 & 20.0 & 0.0 & 5.14 & 1.03\end{array}$

$\begin{array}{cccccc}1.0 & 0.0 & 20.0 & 0.0 & 5.14 & 1.03 \\ 1.0 & 0.0 & 20.0 & 10.0 & 8.34 & 2.47\end{array}$

$\begin{array}{llllll}1.0 & 0.0 & 20.0 & 10.0 & 8.34 & 2.47 \\ 1.0 & 0.0 & 20.0 & 20.0 & 14.41 & 6.40\end{array}$

$\begin{array}{llllll}1.0 & 0.0 & 20.0 & 30.0 & 26.99 & 18.40\end{array}$

$\begin{array}{llllll}1.0 & 0.0 & 20.0 & 30.0 & 26.99 & 18.40 \\ 1.0 & 0.0 & 20.0 & 40.0 & 60.74 & 64.20\end{array}$

$\begin{array}{cccccc}1.0 & 0.0 & 20.0 & 40.0 & 60.74 & 64.20 \\ 1.0 & 0.0 & 30.0 & 0.0 & 5.14 & 1.03\end{array}$

\begin{tabular}{llllll}
1.0 & 0.0 & 30.0 & 10.0 & 8.34 & 2.47 \\
\hline
\end{tabular}


TABle 1: Continued.

\begin{tabular}{|c|c|c|c|c|c|}
\hline$(b / B)$ & $\left(D_{f} / B\right)$ & $\beta$ & $\phi$ & $N^{\prime} c$ & $N^{\prime} q$ \\
\hline 1.0 & 0.0 & 30.0 & 20.0 & 14.23 & 6.40 \\
\hline 1.0 & 0.0 & 30.0 & 30.0 & 25.74 & 18.40 \\
\hline 1.0 & 0.0 & 30.0 & 40.0 & 55.26 & 64.20 \\
\hline 1.0 & 0.0 & 60.0 & 0.0 & 5.14 & 1.03 \\
\hline 1.0 & 0.0 & 60.0 & 10.0 & 8.34 & 2.47 \\
\hline 1.0 & 0.0 & 60.0 & 20.0 & 13.43 & 6.40 \\
\hline 1.0 & 0.0 & 60.0 & 30.0 & 22.46 & 18.40 \\
\hline 1.0 & 0.0 & 60.0 & 40.0 & 43.06 & 64.20 \\
\hline 0.0 & 1.0 & 0.0 & 0.0 & 5.14 & 1.03 \\
\hline 0.0 & 1.0 & 0.0 & 10.0 & 8.34 & 2.47 \\
\hline 0.0 & 1.0 & 0.0 & 20.0 & 14.83 & 6.40 \\
\hline 0.0 & 1.0 & 0.0 & 30.0 & 30.14 & 18.40 \\
\hline 0.0 & 1.0 & 5.0 & 0.0 & 5.14 & 0.99 \\
\hline 0.0 & 1.0 & 5.0 & 10.0 & 8.34 & 2.13 \\
\hline 0.0 & 1.0 & 5.0 & 20.0 & 14.83 & 4.92 \\
\hline 0.0 & 1.0 & 5.0 & 30.0 & 30.14 & 12.55 \\
\hline 0.0 & 1.0 & 5.0 & 40.0 & 75.31 & 38.59 \\
\hline 0.0 & 1.0 & 10.0 & 0.0 & 5.14 & 1.03 \\
\hline 0.0 & 1.0 & 10.0 & 10.0 & 8.34 & 2.19 \\
\hline 0.0 & 1.0 & 10.0 & 20.0 & 14.83 & 4.91 \\
\hline 0.0 & 1.0 & 10.0 & 30.0 & 30.14 & 12.16 \\
\hline 0.0 & 1.0 & 10.0 & 40.0 & 75.31 & 36.24 \\
\hline 0.0 & 1.0 & 20.0 & 0.0 & 5.14 & 1.03 \\
\hline 0.0 & 1.0 & 20.0 & 10.0 & 8.34 & 2.17 \\
\hline 0.0 & 1.0 & 20.0 & 20.0 & 14.83 & 4.63 \\
\hline 0.0 & 1.0 & 20.0 & 30.0 & 30.14 & 10.88 \\
\hline 0.0 & 1.0 & 30.0 & 0.0 & 5.14 & 1.03 \\
\hline 0.0 & 1.0 & 30.0 & 10.0 & 8.34 & 1.89 \\
\hline 0.0 & 1.0 & 30.0 & 20.0 & 14.83 & 4.04 \\
\hline 0.0 & 1.0 & 30.0 & 30.0 & 29.17 & 9.05 \\
\hline 0.0 & 1.0 & 30.0 & 40.0 & 61.39 & 24.02 \\
\hline 0.0 & 1.0 & 60.0 & 0.0 & 5.06 & 0.45 \\
\hline 0.0 & 1.0 & 60.0 & 10.0 & 7.33 & 0.77 \\
\hline 0.0 & 1.0 & 60.0 & 40.0 & 36.93 & 6.27 \\
\hline 0.0 & 0.5 & 0.0 & 0.0 & 5.14 & 1.03 \\
\hline 0.0 & 0.5 & 0.0 & 10.0 & 8.34 & 2.47 \\
\hline 0.0 & 0.5 & 0.0 & 20.0 & 14.83 & 6.40 \\
\hline 0.0 & 0.5 & 0.0 & 30.0 & 30.14 & 18.40 \\
\hline 0.0 & 0.5 & 5.0 & 0.0 & 5.14 & 0.79 \\
\hline 0.0 & 0.5 & 5.0 & 10.0 & 8.34 & 1.69 \\
\hline 0.0 & 0.5 & 5.0 & 20.0 & 14.83 & 4.03 \\
\hline 0.0 & 0.5 & 5.0 & 30.0 & 30.14 & 10.65 \\
\hline 0.0 & 0.5 & 10.0 & 0.0 & 5.14 & 0.79 \\
\hline 0.0 & 0.5 & 10.0 & 10.0 & 8.34 & 1.70 \\
\hline 0.0 & 0.5 & 10.0 & 20.0 & 14.83 & 3.94 \\
\hline 0.0 & 0.5 & 10.0 & 30.0 & 30.14 & 10.15 \\
\hline 0.0 & 0.5 & 20.0 & 0.0 & 5.14 & 0.79 \\
\hline 0.0 & 0.5 & 20.0 & 10.0 & 8.34 & 1.62 \\
\hline 0.0 & 0.5 & 20.0 & 20.0 & 14.83 & 3.58 \\
\hline 0.0 & 0.5 & 20.0 & 30.0 & 28.19 & 8.79 \\
\hline 0.0 & 0.5 & 30.0 & 0.0 & 5.14 & 0.72 \\
\hline 0.0 & 0.5 & 30.0 & 10.0 & 8.34 & 1.43 \\
\hline 0.0 & 0.5 & 30.0 & 20.0 & 13.83 & 3.03 \\
\hline 0.0 & 0.5 & 30.0 & 30.0 & 25.11 & 7.09 \\
\hline 0.0 & 0.5 & 60.0 & 0.0 & 4.34 & 0.28 \\
\hline 0.0 & 0.5 & 60.0 & 10.0 & 6.33 & 0.50 \\
\hline 0.0 & 0.5 & 60.0 & 20.0 & 9.81 & 0.95 \\
\hline 0.0 & 0.5 & 60.0 & 30.0 & 16.68 & 1.98 \\
\hline 0.5 & 1.0 & 5.0 & 0.0 & 5.14 & 1.03 \\
\hline 0.5 & 1.0 & 5.0 & 10.0 & 8.34 & 2.25 \\
\hline
\end{tabular}

TABle 1: Continued.

\begin{tabular}{|c|c|c|c|c|c|}
\hline$(b / B)$ & $\left(D_{f} / B\right)$ & $\beta$ & $\phi$ & $N^{\prime} c$ & $N^{\prime} q$ \\
\hline 0.5 & 1.0 & 5.0 & 20.0 & 14.83 & 5.19 \\
\hline 0.5 & 1.0 & 5.0 & 30.0 & 30.14 & 13.22 \\
\hline 0.5 & 1.0 & 5.0 & 40.0 & 75.31 & 40.59 \\
\hline 0.5 & 1.0 & 10.0 & 0.0 & 5.14 & 1.03 \\
\hline 0.5 & 1.0 & 10.0 & 10.0 & 8.34 & 2.44 \\
\hline 0.5 & 1.0 & 10.0 & 20.0 & 14.83 & 5.47 \\
\hline 0.5 & 1.0 & 10.0 & 30.0 & 30.14 & 13.52 \\
\hline 0.5 & 1.0 & 10.0 & 40.0 & 75.31 & 40.14 \\
\hline 0.5 & 1.0 & 20.0 & 0.0 & 5.14 & 1.03 \\
\hline 0.5 & 1.0 & 20.0 & 10.0 & 8.34 & 2.47 \\
\hline 0.5 & 1.0 & 20.0 & 20.0 & 14.83 & 5.80 \\
\hline 0.5 & 1.0 & 20.0 & 30.0 & 30.14 & 13.56 \\
\hline 0.5 & 1.0 & 20.0 & 40.0 & 73.61 & 37.73 \\
\hline 0.5 & 1.0 & 30.0 & 0.0 & 5.14 & 1.03 \\
\hline 0.5 & 1.0 & 30.0 & 10.0 & 8.34 & 2.47 \\
\hline 0.5 & 1.0 & 30.0 & 20.0 & 14.83 & 5.77 \\
\hline 0.5 & 1.0 & 30.0 & 30.0 & 30.14 & 12.81 \\
\hline 0.5 & 1.0 & 30.0 & 40.0 & 65.57 & 33.55 \\
\hline 0.5 & 1.0 & 60.0 & 0.0 & 5.14 & 1.03 \\
\hline 0.5 & 1.0 & 60.0 & 10.0 & 8.34 & 2.04 \\
\hline 0.5 & 1.0 & 60.0 & 20.0 & 14.83 & 3.63 \\
\hline 0.5 & 1.0 & 60.0 & 30.0 & 23.09 & 7.01 \\
\hline 0.5 & 1.0 & 60.0 & 40.0 & 44.19 & 15.55 \\
\hline 1.0 & 1.0 & 5.0 & 0.0 & 5.14 & 1.03 \\
\hline 1.0 & 1.0 & 5.0 & 10.0 & 8.34 & 2.34 \\
\hline 1.0 & 1.0 & 5.0 & 20.0 & 14.83 & 5.41 \\
\hline 1.0 & 1.0 & 5.0 & 30.0 & 30.14 & 13.81 \\
\hline 1.0 & 1.0 & 5.0 & 40.0 & 75.31 & 42.45 \\
\hline 1.0 & 1.0 & 10.0 & 0.0 & 5.14 & 1.03 \\
\hline 1.0 & 1.0 & 10.0 & 10.0 & 8.34 & 2.47 \\
\hline 1.0 & 1.0 & 10.0 & 20.0 & 14.83 & 5.95 \\
\hline 1.0 & 1.0 & 10.0 & 30.0 & 30.14 & 14.75 \\
\hline 1.0 & 1.0 & 10.0 & 40.0 & 75.31 & 43.83 \\
\hline 1.0 & 1.0 & 20.0 & 0.0 & 5.14 & 1.03 \\
\hline 1.0 & 1.0 & 20.0 & 10.0 & 8.34 & 2.47 \\
\hline 1.0 & 1.0 & 20.0 & 20.0 & 14.83 & 6.40 \\
\hline 1.0 & 1.0 & 20.0 & 30.0 & 30.14 & 16.11 \\
\hline 1.0 & 1.0 & 20.0 & 40.0 & 75.31 & 44.80 \\
\hline 1.0 & 1.0 & 30.0 & 0.0 & 5.14 & 1.03 \\
\hline 1.0 & 1.0 & 30.0 & 10.0 & 8.34 & 2.47 \\
\hline 1.0 & 1.0 & 30.0 & 20.0 & 14.83 & 6.40 \\
\hline 1.0 & 1.0 & 30.0 & 30.0 & 30.14 & 16.11 \\
\hline 1.0 & 1.0 & 30.0 & 40.0 & 69.76 & 43.38 \\
\hline 1.0 & 1.0 & 60.0 & 0.0 & 5.14 & 1.03 \\
\hline 1.0 & 1.0 & 60.0 & 10.0 & 8.34 & 2.47 \\
\hline 1.0 & 1.0 & 60.0 & 20.0 & 14.83 & 6.36 \\
\hline 1.0 & 1.0 & 60.0 & 30.0 & 27.14 & 12.28 \\
\hline 1.0 & 1.0 & 60.0 & 40.0 & 51.44 & 27.10 \\
\hline 0.5 & 1.5 & 5.0 & 0.0 & 5.14 & 1.03 \\
\hline 0.5 & 1.5 & 5.0 & 10.0 & 8.34 & 2.47 \\
\hline 0.5 & 1.5 & 5.0 & 20.0 & 14.83 & 6.02 \\
\hline 0.5 & 1.5 & 5.0 & 30.0 & 30.14 & 14.95 \\
\hline 0.5 & 1.5 & 10.0 & 0.0 & 5.14 & 1.03 \\
\hline 0.5 & 1.5 & 10.0 & 10.0 & 8.34 & 2.47 \\
\hline 0.5 & 1.5 & 10.0 & 20.0 & 14.83 & 6.33 \\
\hline 0.5 & 1.5 & 10.0 & 30.0 & 30.14 & 15.26 \\
\hline 0.5 & 1.5 & 10.0 & 40.0 & 75.31 & 43.94 \\
\hline 0.5 & 1.5 & 20.0 & 0.0 & 5.14 & 1.03 \\
\hline 0.5 & 1.5 & 20.0 & 10.0 & 8.34 & 2.47 \\
\hline 0.5 & 1.5 & 20.0 & 20.0 & 14.83 & 6.40 \\
\hline
\end{tabular}


Table 1: Continued.

\begin{tabular}{|c|c|c|c|c|c|}
\hline$(b / B)$ & $\left(D_{f} / B\right)$ & $\beta$ & $\phi$ & $N^{\prime} c$ & $N^{\prime} q$ \\
\hline 0.5 & 1.5 & 20.0 & 30.0 & 30.14 & 15.20 \\
\hline 0.5 & 1.5 & 20.0 & 40.0 & 75.31 & 41.04 \\
\hline 0.5 & 1.5 & 30.0 & 0.0 & 5.14 & 1.03 \\
\hline 0.5 & 1.5 & 30.0 & 10.0 & 8.34 & 2.47 \\
\hline 0.5 & 1.5 & 30.0 & 20.0 & 14.83 & 6.40 \\
\hline 0.5 & 1.5 & 30.0 & 30.0 & 30.14 & 14.23 \\
\hline 0.5 & 1.5 & 30.0 & 40.0 & 72.83 & 36.18 \\
\hline 0.5 & 1.5 & 60.0 & 0.0 & 5.14 & 1.03 \\
\hline 0.5 & 1.5 & 60.0 & 10.0 & 8.34 & 2.19 \\
\hline 0.5 & 1.5 & 60.0 & 20.0 & 14.83 & 3.85 \\
\hline 0.5 & 1.5 & 60.0 & 30.0 & 25.43 & 7.28 \\
\hline 0.5 & 1.5 & 60.0 & 40.0 & 48.37 & 15.77 \\
\hline 0.0 & 1.5 & 0.0 & 0.0 & 5.14 & 1.03 \\
\hline 0.0 & 1.5 & 0.0 & 10.0 & 8.34 & 2.47 \\
\hline 0.0 & 1.5 & 0.0 & 20.0 & 14.83 & 6.40 \\
\hline 0.0 & 1.5 & 0.0 & 30.0 & 30.14 & 18.40 \\
\hline 0.0 & 1.5 & 5.0 & 0.0 & 5.14 & 1.03 \\
\hline 0.0 & 1.5 & 5.0 & 10.0 & 8.34 & 2.47 \\
\hline 0.0 & 1.5 & 5.0 & 20.0 & 14.83 & 5.79 \\
\hline 0.0 & 1.5 & 5.0 & 30.0 & 30.14 & 14.40 \\
\hline 0.0 & 1.5 & 10.0 & 0.0 & 5.14 & 1.03 \\
\hline 0.0 & 1.5 & 10.0 & 10.0 & 8.34 & 2.47 \\
\hline 0.0 & 1.5 & 10.0 & 20.0 & 14.83 & 5.85 \\
\hline 0.0 & 1.5 & 10.0 & 30.0 & 30.14 & 14.13 \\
\hline 0.0 & 1.5 & 20.0 & 0.0 & 5.14 & 1.03 \\
\hline 0.0 & 1.5 & 20.0 & 10.0 & 8.34 & 2.47 \\
\hline 0.0 & 1.5 & 20.0 & 20.0 & 14.83 & 5.65 \\
\hline 0.0 & 1.5 & 20.0 & 30.0 & 30.14 & 12.93 \\
\hline 0.0 & 1.5 & 20.0 & 40.0 & 75.31 & 35.14 \\
\hline 0.0 & 1.5 & 30.0 & 0.0 & 5.14 & 1.03 \\
\hline 0.0 & 1.5 & 30.0 & 10.0 & 8.34 & 2.47 \\
\hline 0.0 & 1.5 & 30.0 & 20.0 & 14.83 & 5.04 \\
\hline 0.0 & 1.5 & 30.0 & 30.0 & 30.14 & 10.99 \\
\hline 0.0 & 1.5 & 30.0 & 40.0 & 68.64 & 28.23 \\
\hline 0.0 & 1.5 & 60.0 & 0.0 & 5.14 & 0.62 \\
\hline 0.0 & 1.5 & 60.0 & 10.0 & 8.34 & 1.04 \\
\hline 1.0 & 1.5 & 5.0 & 0.0 & 5.14 & 1.03 \\
\hline 1.0 & 1.5 & 5.0 & 10.0 & 8.34 & 2.47 \\
\hline 1.0 & 1.5 & 5.0 & 20.0 & 14.83 & 6.21 \\
\hline 1.0 & 1.5 & 5.0 & 30.0 & 30.14 & 15.45 \\
\hline 1.0 & 1.5 & 10.0 & 0.0 & 5.14 & 1.03 \\
\hline 1.0 & 1.5 & 10.0 & 10.0 & 8.34 & 2.47 \\
\hline 1.0 & 1.5 & 10.0 & 20.0 & 14.83 & 6.40 \\
\hline 1.0 & 1.5 & 10.0 & 30.0 & 30.14 & 16.30 \\
\hline 1.0 & 1.5 & 10.0 & 40.0 & 75.31 & 46.93 \\
\hline 1.0 & 1.5 & 20.0 & 0.0 & 5.14 & 1.03 \\
\hline 1.0 & 1.5 & 20.0 & 10.0 & 8.34 & 2.47 \\
\hline 1.0 & 1.5 & 20.0 & 20.0 & 14.83 & 6.40 \\
\hline 1.0 & 1.5 & 20.0 & 30.0 & 30.14 & 17.48 \\
\hline 1.0 & 1.5 & 20.0 & 40.0 & 75.31 & 46.85 \\
\hline 1.0 & 1.5 & 30.0 & 0.0 & 5.14 & 1.03 \\
\hline 1.0 & 1.5 & 30.0 & 10.0 & 8.34 & 2.47 \\
\hline 1.0 & 1.5 & 30.0 & 20.0 & 14.83 & 6.40 \\
\hline 1.0 & 1.5 & 30.0 & 30.0 & 30.14 & 17.48 \\
\hline 1.0 & 1.5 & 30.0 & 40.0 & 75.31 & 44.32 \\
\hline 1.0 & 1.5 & 60.0 & 0.0 & 5.14 & 1.03 \\
\hline 1.0 & 1.5 & 60.0 & 10.0 & 8.34 & 2.47 \\
\hline 1.0 & 1.5 & 60.0 & 20.0 & 14.83 & 6.17 \\
\hline 1.0 & 1.5 & 60.0 & 30.0 & 29.49 & 11.69 \\
\hline 1.0 & 1.5 & 60.0 & 40.0 & 55.63 & 25.26 \\
\hline
\end{tabular}

TABLE 2: Statistical analysis of the generated database.

\begin{tabular}{lccccc}
\hline & Minimum & Maximum & Mean & Std. deviation & Variance \\
\hline$b / B$ & 0.00 & 1.00 & 0.492 & 0.412 & 0.170 \\
$D_{f} / B$ & 0.00 & 1.50 & 0.735 & 0.561 & 0.315 \\
$\beta$ & 0.00 & 60.00 & 23.217 & 19.403 & 376.491 \\
$\phi$ & 0.00 & 40.00 & 18.900 & 13.848 & 191.763 \\
$N^{\prime} c$ & 3.62 & 75.31 & 21.972 & 21.100 & 445.201 \\
$N^{\prime} q$ & 0.28 & 64.20 & 12.204 & 16.766 & 281.101 \\
\hline
\end{tabular}

TABle 3: Pearson correlation matrix.

\begin{tabular}{lcccccc}
\hline & $b / B$ & $D_{f} / B$ & $\beta$ & $\phi$ & $N^{\prime} c$ & $N^{\prime} q$ \\
\hline$b / B$ & 1.000 & & & & & \\
$D_{f} / B$ & 0.023 & 1.000 & & & & \\
$\beta$ & 0.119 & -0.011 & 1.000 & & & \\
$\phi$ & 0.069 & -0.031 & 0.008 & 1.000 & & \\
$N^{\prime} c$ & 0.104 & 0.023 & -0.090 & 0.867 & 1.000 & \\
$N^{\prime} q$ & 0.105 & -0.166 & -0.044 & 0.787 & 0.889 & 1.000 \\
\hline
\end{tabular}

3.1.3. Model (3)-Using the EPR Technique. Finally, the developed EPR models were limited to the pentagonal level, for 4 inputs; there are 226 possible terms $(56+35+20+10+4+1=126)$ as follows:

$$
\begin{aligned}
\sum_{i=1}^{i=4} \sum_{j=1}^{j=4} \sum_{k=1}^{k=4} \sum_{l=1}^{l=4} \sum_{m=1}^{m=4} X_{i} \cdot X_{j} \cdot X_{k} \cdot X_{l} \cdot X_{m} & +\sum_{i=1}^{i=4} \sum_{j=1}^{j=4} \sum_{k=1}^{k=4} \sum_{l=1}^{l=4} X_{i} \cdot X_{j} \cdot X_{k} \cdot X_{l} \\
& +\sum_{i=1}^{i=4} \sum_{j=1}^{j=4} \sum_{k=1}^{k=4} X_{i} \cdot X_{j} \cdot X_{k} \\
& +\sum_{i=1}^{i=4} \sum_{j=1}^{j=4} X_{i} \cdot X_{j}+\sum_{i=1}^{i=4} X_{i}+C .
\end{aligned}
$$

The GA technique was applied on these 126 terms to select the most effective 6 terms to predict the values of $N^{\prime} c$ and $N^{\prime} q$. The output is illustrated in equations (3) and (4), and their fitness is shown in Figures 3(c) and 4(c). The average errors (\%) and $R^{2}$ values were $7.2 \%-0.994$ for $N^{\prime} c$ and $17.3 \%-0.984$ for $N^{\prime} q$. Both equations (5) and (6) show that $\phi$ existed in each term and it got the highest power, which indicated that it is the most effective parameter, and other parameters almost had the same importance. Meanwhile, the results of all the developed models are summarized in Tables 5 and 6 .

$$
\begin{aligned}
N^{\prime} c= & \frac{\phi^{4}}{45000}+\frac{\phi}{4 \cdot 1}+\frac{b \cdot \beta \cdot \phi}{205 B}+\frac{D_{f} \cdot \phi^{3}}{8250 B}-\frac{\beta \cdot \phi^{3}}{98000}+5.165 \\
N^{\prime} q= & \frac{\phi^{4}}{43350}+\frac{D_{f}^{2} \cdot \phi^{3}}{2140 B^{2}}+\frac{D_{f} \cdot b \cdot \beta \cdot \phi^{2}}{4170 B^{2}}-\frac{D_{f} \cdot \phi^{3}}{1335 B} \\
& -\frac{D_{f} \cdot \beta \cdot \phi^{3}}{100000 B}+2 \cdot 253 .
\end{aligned}
$$



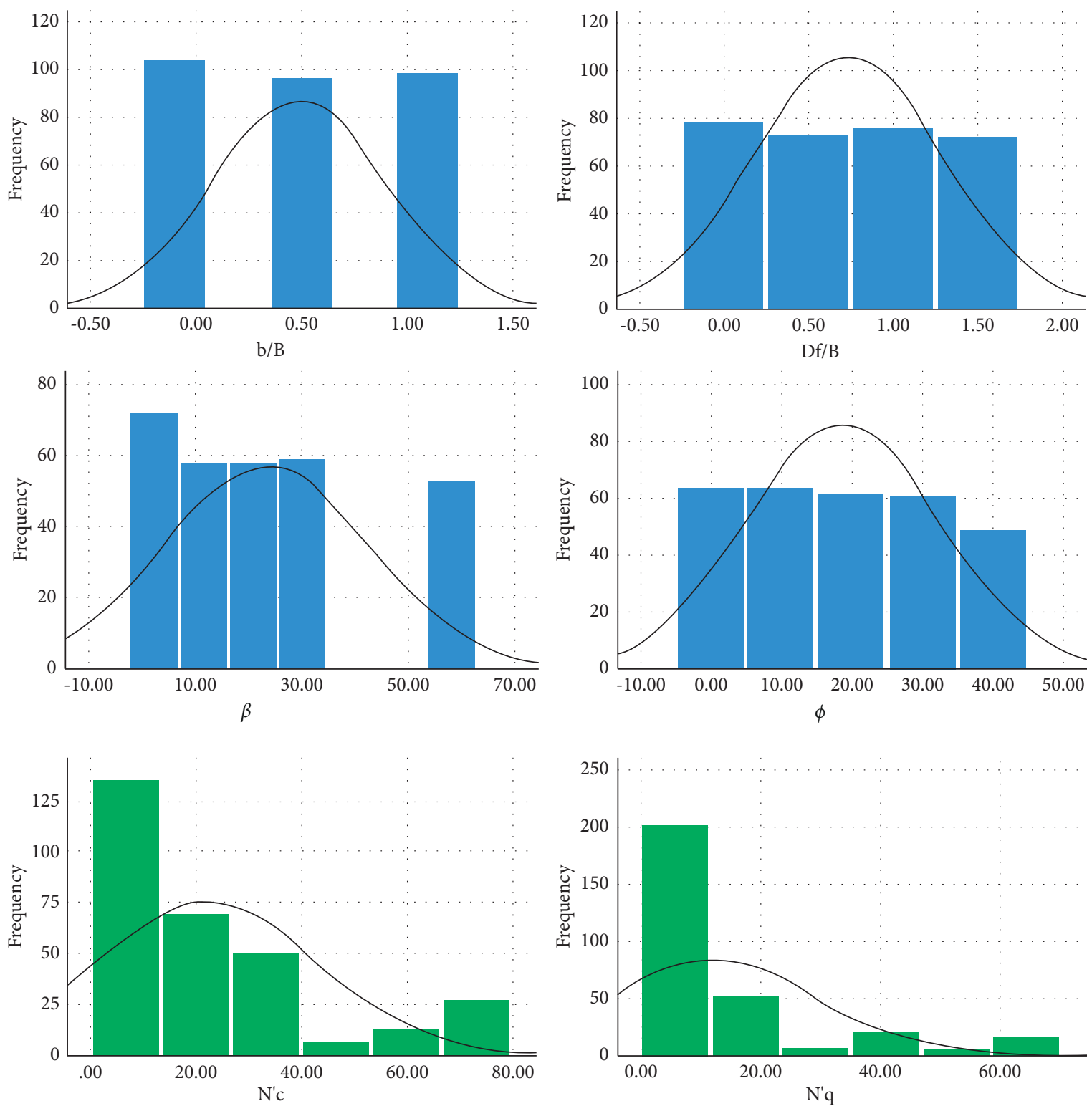

FIGURE 2: Distribution histograms for inputs (in blue) and outputs (in green).

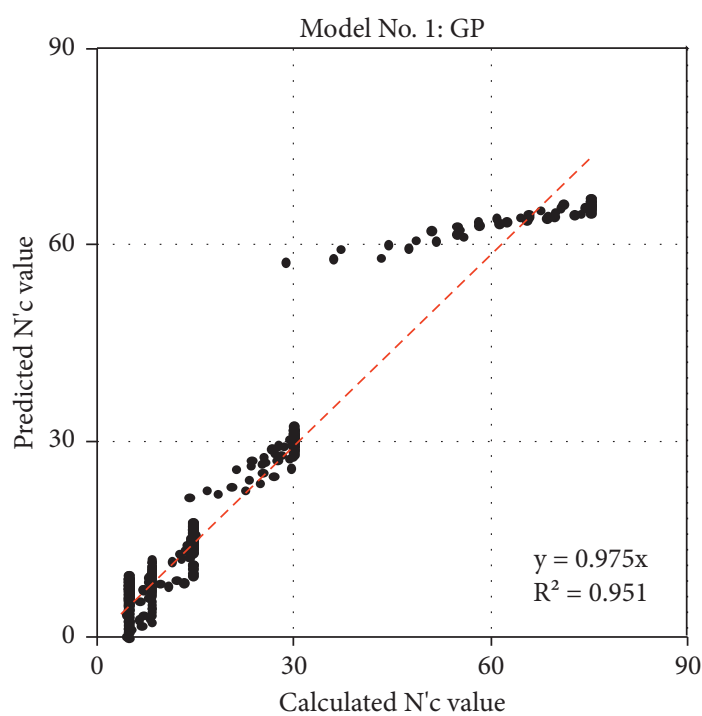

(a)

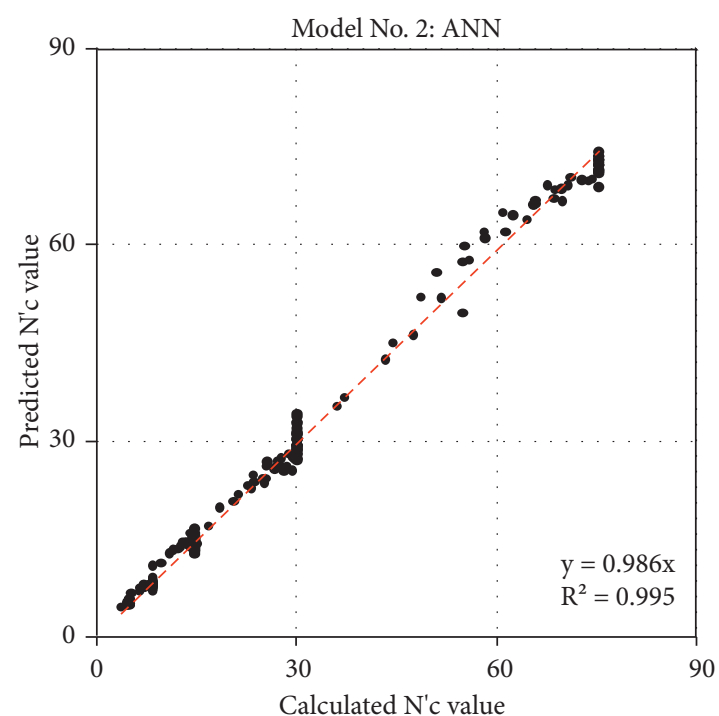

(b)

Figure 3: Continued. 


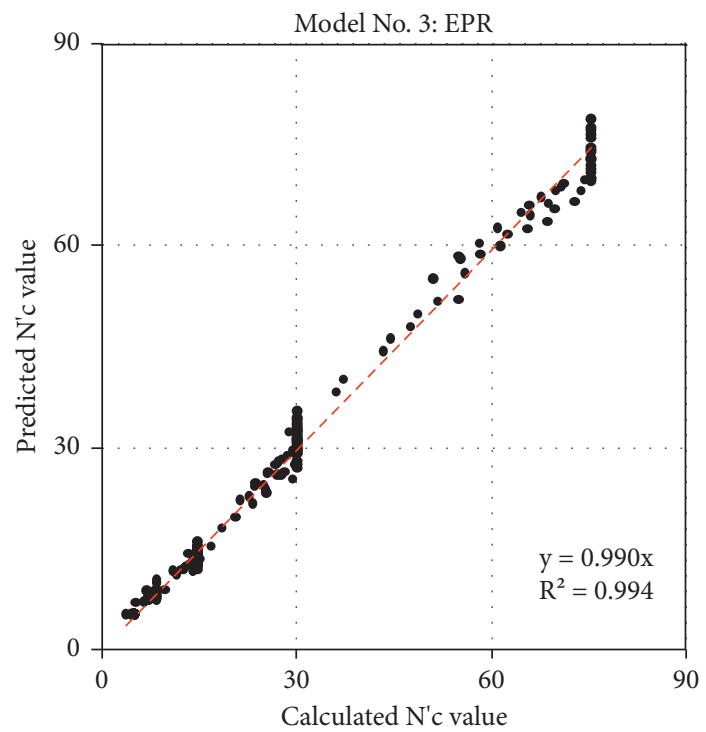

(c)

FIGURE 3: Relation between the predicted and calculated $N^{\prime} c$ values using the developed (a) GP, (b) ANN, and (c) EPR models.

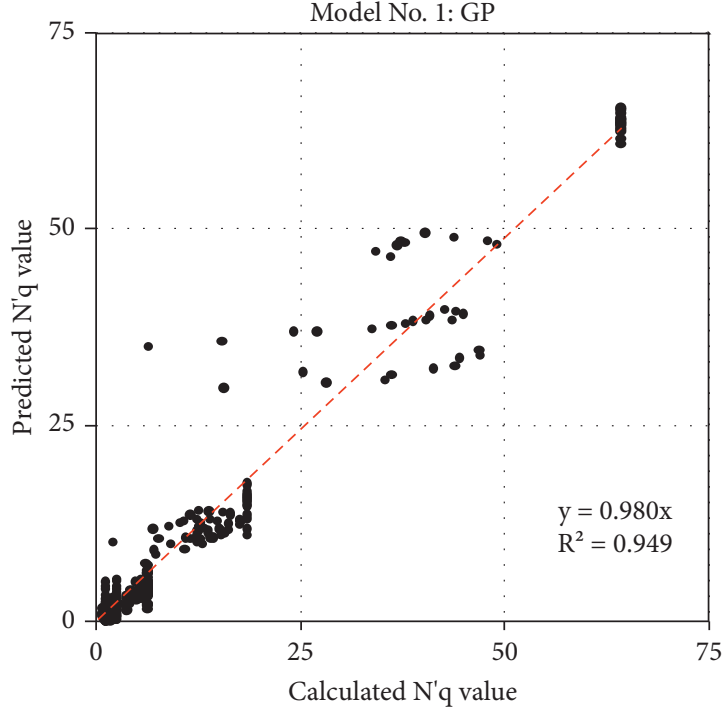

(a)

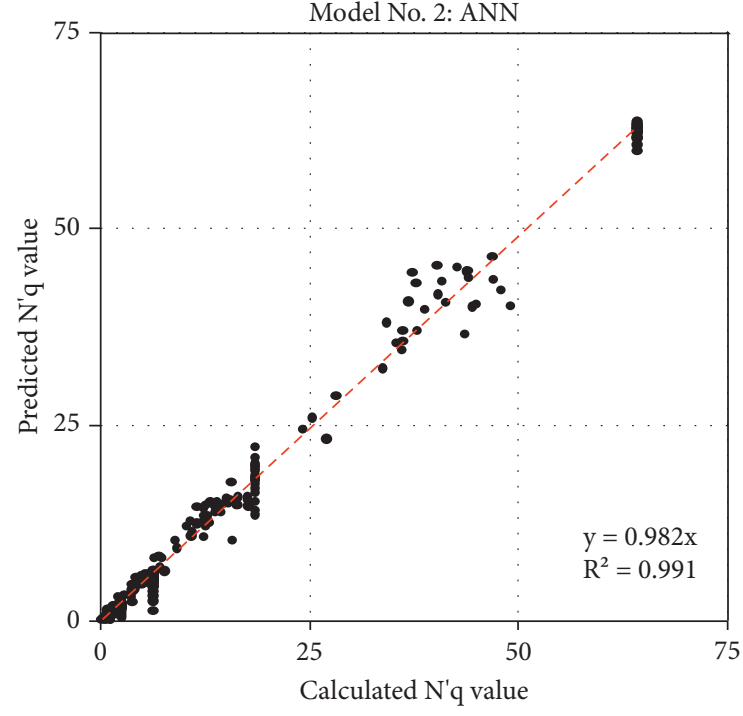

(b)

Figure 4: Continued. 


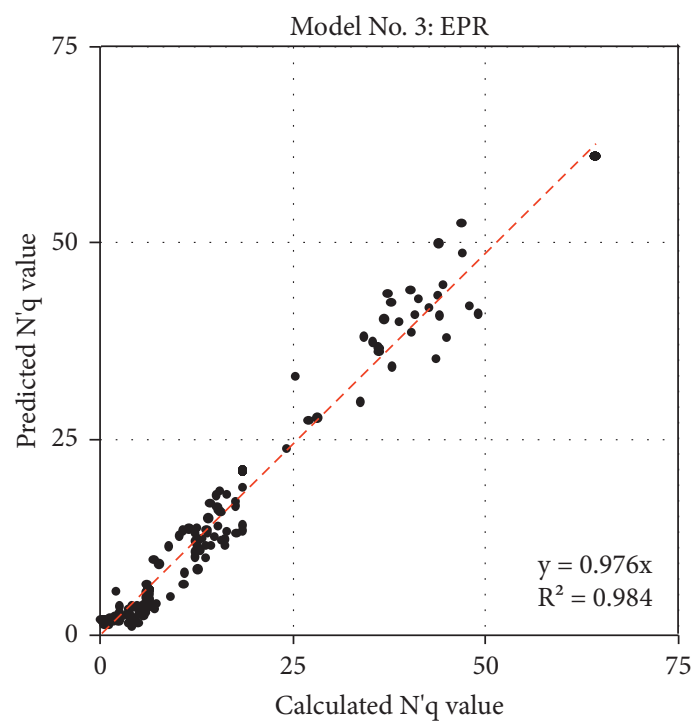

(c)

FIGURE 4: Relation between the predicted and calculated $N^{\prime} q$ values using the developed (a) GP, (b) ANN, and (c) EPR models.

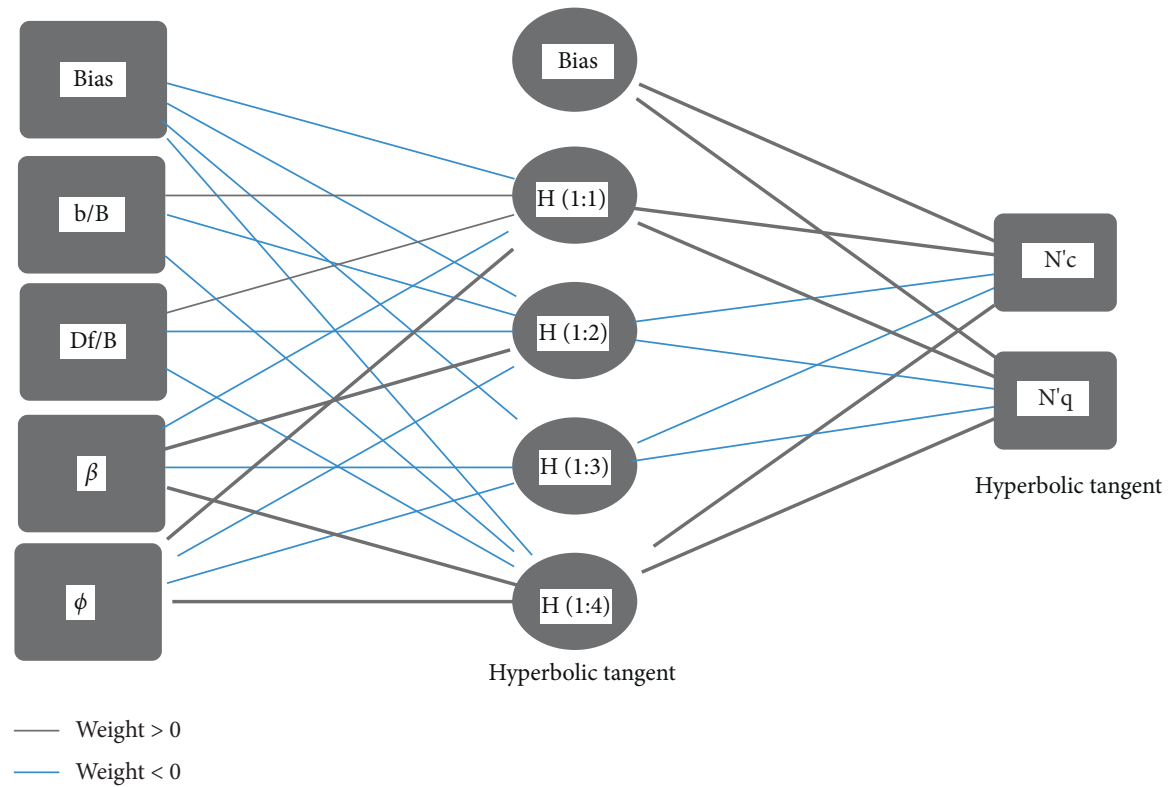

FIgURE 5: Layout for the developed ANN.

TABLE 4: Connection weights of the developed ANN model.

\begin{tabular}{|c|c|c|c|c|c|c|c|}
\hline \multirow{2}{*}{\multicolumn{2}{|c|}{ Predictor }} & \multicolumn{4}{|c|}{ Hidden layer 1} & \multicolumn{2}{|c|}{ Output layer } \\
\hline & & $H(1: 1)$ & $H(1: 2)$ & $H(1: 3)$ & $H(1: 4)$ & $N^{\prime} c$ & $N^{\prime} q$ \\
\hline \multirow{5}{*}{ Input layer } & (Bias) & -3.177 & -0.836 & -0.387 & -3.987 & & \\
\hline & $b / B$ & 0.085 & -0.333 & 0.077 & -0.177 & & \\
\hline & $D_{f} / B$ & 0.237 & -0.185 & 0.035 & -1.768 & & \\
\hline & $\beta$ & -0.511 & 0.563 & -0.187 & 0.415 & & \\
\hline & $\phi$ & 2.644 & -0.109 & -0.750 & 2.130 & & \\
\hline \multirow{5}{*}{ Hidden layer 1} & (Bias) & & & & & 0.365 & 0.592 \\
\hline & $\mathrm{H}(1: 1)$ & & & & & 2.027 & 0.730 \\
\hline & $H(1: 2)$ & & & & & -0.608 & -1.372 \\
\hline & $H(1: 3)$ & & & & & -1.283 & -1.470 \\
\hline & $H(1: 4)$ & & & & & 0.053 & 2.560 \\
\hline
\end{tabular}


TABle 5: Accuracies of the developed models for $N^{\prime} c$.

\begin{tabular}{lccc}
\hline Technique & Developed equation & Error (\%) & $R^{2}$ \\
\hline GP & Equation (2) & 20.7 & 0.951 \\
ANN & Table 4 & 6.9 & 0.995 \\
EPR & Equation (4) & 7.2 & 0.994 \\
\hline
\end{tabular}

TABle 6: Accuracies of the developed models for $N^{\prime} q$.

\begin{tabular}{lccc}
\hline Technique & Developed equation & Error (\%) & $R^{2}$ \\
\hline GP & Equation (3) & 31.6 & 0.949 \\
ANN & Table 4 & 13.0 & 0.991 \\
EPR & Equation (5) & 17.3 & 0.984 \\
\hline
\end{tabular}

\section{Conclusions}

This research presents three models using three AI techniques, namely, GP, ANN, and EPR, to predict the modified bearing capacity parameters $N^{\prime} c$ and $N^{\prime} q$ using slope angle $(\beta)$, angle of internal friction of soil $(\phi)$, ratio between the distance to the slope edge and footing width $(b / B)$, and the ratio between foundation depth and footing width $\left(D_{f} / B\right)$. The results of comparing the accuracies of the developed models could be concluded in the following points:

(i) Although five levels of complexity (128 genes in the chromosome) were used in the GP model, the achieved accuracy was still low (79.3\% and 68.4\% for $N^{\prime} c$ and $N^{\prime} q$, respectively)

(ii) The prediction accuracies of ANN and EPR models are close, $93.1 \%$ and $92.8 \%$ for $N^{\prime} c$ and $87.0 \%$ and $82.7 \%$ for $N^{\prime} q$, which gives an advantage to the EPR model because its output is a simple equation and could be applied either manually or implemented in software unlike the complicated output of the ANN which cannot be applied manually

(iii) The importance analysis of both ANN and EPR models indicated that $\left(N^{\prime} c\right.$ and $\left.N^{\prime} q\right)$ values were mainly governed by $\phi$, while other parameters are minors

(iv) All developed models showed that $N^{\prime} c$ and $N^{\prime} q$ values increase with increasing $\phi, b / B$, and $D_{f} / B$ values and with decreasing $\beta$ value

(v) GA technique successfully reduced the 126 terms of the conventional PLR quadratic formula to only 6 terms without a significant impact on its accuracy

(vi) Like any other regression technique, the generated formulas are valid within the considered range of parameter values; beyond this range, the prediction accuracy should be verified

\section{Abbreviations}

AI: Artificial intelligence

C: Cohesion

q: $\quad$ Overburden pressure at foundation depth

$\gamma$ : $\quad$ Bulk density of soil below footing

B: $\quad$ Strip footing width
L0: Length of the failure wedge of normal footing

L1: $\quad$ Length of the failure wedge of slopy footing

A0: $\quad$ Area of a rectangular mass on normal footing

A1: Area of a triangular mass on slopy footing

$b$ : Distance of the slope from the footing edge

$\beta$ : $\quad$ Slope angle

$\phi$ : Friction

Nc: Bearing capacity coefficient of cohesion

$N q$ : Bearing capacity coefficient of overburden pressure

$N \gamma$ : $\quad$ Bearing capacity coefficient of bulk density

$N^{\prime} c$ : Predicted bearing capacity coefficient of cohesion on the slope

$N^{\prime} q$ : Predicted bearing capacity coefficient of overburden pressure on the slope

ANN: Artificial neural network

GP: Genetic programming'

GA: Genetic algorithm

EPR: Evolutionary polynomial regression

PLR: Polynomial linear regression

SSE: Sum of squared errors

$R^{2}$ : Coefficient of determination.

\section{Data Availability}

The underlying data supporting the results of this research are reported in the manuscript.

\section{Conflicts of Interest}

The authors declare no conflicts of interest in the publication of this research work.

\section{References}

[1] R. Acharyya and A. Dey, "Assessment of interaction mechanism and bearing capacity of strip footings located on slope face," Sadhana-Academy Proceedings in Engineering Sciences, vol. 45, no. 89, 2020.

[2] O. Casablanca, G. Biondi, E. Cascone, and G. D. Filippo, "Static and seismic bearing capacity of shallow strip foundations," Géotechnique, vol. 20, no. 44, 2021.

[3] D. Shaunik and M. Singh, "Bearing capacity of foundation on rock slopes intersected by non-persistent discontinuity," International Journal of mining Sciences and Technology, vol. 30, no. 5, pp. 669-674, 2020.

[4] S. Yang, B. Leshchinsky, and K. Gui, F. Zhang and Q. Feng, Influence of failure mechanisms on seismic bearing capacity factors for shallow foundations near slopes," Géotechnique, vol. 71, no. 7, pp. 594-607, 2021.

[5] H. Zhou, G. Zheng, X. Yang, T. Li, and P. Yang, "Ultimate seismic bearing capacities and failure mechanisms for strip footings placed adjacent to slopes," Canadian Geotechnical Journal, vol. 56, no. 11, pp. 1729-1735, 2019.

[6] Y. Jiang, W. Gu, and J. Chen, "Analysis of ultimate bearing capacity factor of buried strip foundation adjacent to slope based on Terzaghi theory of foundation ultimate bearing capacity," Fresenius Environmental Bulletin, vol. 30, no. 5, 2021.

[7] T. Mansouri and K. Abbeche, "Experimental bearing capacity of eccentrically loaded foundation near a slope," Studia Geotechnica et Mechanica, vol. 41, no. 1, pp. 33-41, 2019. 
[8] D. Raj and M. Bharathi, "Bearing capacity of shallow foundation on slope: a review," in Proceedings of the 4th ICSMFE, London, UK, February 2013.

[9] S. Yang, B. Leshchinksky, K. Cui, F. Zhang, and Y. Gao, "Unified approach towards evaluating bearing capacity of shallow foundations near slopes," Journal of Geotechnical and Geoenvironmental Engineering, vol. 145, no. 2, 2019.

[10] A. Foroutan Kalourazi and A. Izadi, R. J. Chenari, Siesmic bearing capacity of shallow strip foundation in the vicinity of slopes using the lower bound finite element method," Soils and founations, vol. 59, no. 6, pp. 1891-1905, 2019.

[11] S. Li, Y. Liu, C. Jang, Y. Cao, and F. Peng, "Study of upper limit solution and its application on bearing of rock slope foundation," IOP Conference Series: Earth and Environmental Science, vol. 560, pp. 23-26, 2020.

[12] H. Javdanian, "On the behaviour of shallow foundations constructed on reinforced soil slope-a numerical analysis," Geotechnical Engineering, vol. 14, no. 2, pp. 188-195, 2020.

[13] K. C. Onyelowe and J. C. Agunwamba, "Variational solution of critical normal stress distribution of footing on slope," Journal of Emerging Trends in Engineering and Applied Sciences, vol. 2, no. 5, pp. 826-834, 2011.

[14] C. Li, Y. Guan, P. Jiang, and X. Han, "Oblique bearing capacity of shallow foundations placed near slopes determined by the mrthod rigorous characteristics," Géotechnique, vol. 20, p. $355,2021$.

[15] J. Liangxing, Y. Feng, H. Zhang, and Q. Feng, "The use of improved movement optimization to calculate the ultimate bearing capacity of a non-homogenous clay foundation adjacent to slopes," Computers and Geotechnics, vol. 118, Article ID 103338, 2000.

[16] R. Acharyya and A. Dey, "Finite element investigation of the bearing capacity of square footings resting on sloping ground," INAE Letters, vol. 2, no. 3, pp. 97-105, 2017.

[17] B. S. Van, "The bearing capacity of shallow foundation on slopes," Numerical Methods in Geotechnical Engineering, vol. IX, 2019.

[18] L. Prandtl, "Uber die eindringungs-festigkeit (harte) plastischer baustoffe und die festigkeit von schneiden," Zeitschrift fur Angewandte Mathematik und Mechanik, vol. 1, pp. 15-20, 1921.

[19] K. Terzaghi, Die Berechung der Durchlaessigkeitsziffer des Toneseausdem Varlauf der Hydrodynamischenspannugserschinungeen, Vol. 132, Sitzungsberichte de Akadennie der wissehsahaften Abt., II a, , Vienna, Austria, 1943.

[20] G. G. Meyerhof, "Ultimate bearing capacity of footings on sand layer overlying clay," Canadian Geotechnical Journal, vol. 11, pp. 223-229, 1974.

[21] A. S. Vesic, Foundation Engineering HandbookVan Nostrand Reinhold Co., New York, NY, USA, 1975.

[22] G. G. Meyerhof, "The ultimate bearing capacity of foundations on slopes," in Proceedings of the 4th International Conference on Soil Mechanics and Foundation Engineering, vol. 3, pp. 384-386, London, UK, 1957.

[23] J. B. Hansen, A Revised and Extended Formula for Bearing Capacity, Danish Geotechnical Institute, Copenhagen, Denmark, 1970.

[24] G. Graham, M. Andrews, and D. H. Shields, "Stress characteristics for shallow footings in cohesionless slopes," $\mathrm{Ca}$ nadian Geotechnical Journal, vol. 25, no. 2, pp. 238-249, 1988.

[25] D. V. Griffiths, "Computation of bearing capacity factors using finite elements," Géotechnique, vol. 32, no. 3, pp. 195-202, 1982.
[26] O. Kusakabe, T. Kimura, and H. Yamaguchi, "Bearing Capacity of slopes under strip loads on the top surfaces, soils found," JGS, vol. 21, no. 4, pp. 29-40, 1981.

[27] B. Leshchinsky, "Bearing capacity of footings placed adjacent to C- $\phi$ slopes," Journal of Geotechnical and Geoenvironmental Engineering, vol. 1, no. 1, pp. 1-20, 2015.

[28] H. Moayedi and A. Rezaei, "The feasibility of PSO-ANFIS in estimating bearing capacity of strip foundation rested on cohesionless slope," Neural Computing \& Applications, vol. 33, pp. 4165-4177, 2021.

[29] A. M. Ebid, "35 years of (AI) in geotechnical engineering: state of the art," Geotechnical \& Geological Engineering, vol. 39, no. 2, pp. 637-690, 2020.

[30] A. A. Gholampour, A. H. Gandomi, and T. Ozbakkaloghu, "New formulations for mechanics properties of recycled aggregate concrete using gene expression programming," Construction and Building Materials, vol. 130, pp. 122-145, 2017.

[31] K. C. Onyelowe and J. Shakeri, "Intelligent prediction of coefficients of curvature and uniformity of hybrid cement modified unsaturated soil with NQF inclusion," Cleaner Engineering and Technology, vol. 4, 2021.

[32] G. Tayfur, "Modern optimization methods in water resources planning, engineering and management," Water Resources Management, vol. 31, no. 10, pp. 3205-3233, 2017.

[33] E. Momeni, D. J. Armaghani, S. A. Fatemi, and R. Nazir, "Prediction of bearing capacity of thin-walled foundation: a simulation approach," Engineering with Computers, vol. 34, no. 2, pp. 319-327, 2018.

[34] F. J. Muhammad, N. A. Muhammad, I. S. Muhammad et al., "Applications of gene expression programming and regression techniques for estimating compressive strength of bagasse ash-based concrete," Crystals, vol. 10, no. 373, pp. 1-17, 2020.

[35] K. C. Onyelowe, A. Ebid, L. Nwobia, and L. Dao-Phuc, "Prediction and performance analysis of compression index of multiple-binder treated soil by genetic programming approach," Nanotechnology for Environmental Engineering, vol. 6, p. 28, 2021.

[36] K. C. Onyelowe, M. Iqbal, F. Jalal, M. Onyia, and I. Onuoha, "Application of 3 algorithm ANN programming to predict the strength performance of hydrated-lime activated rice husk ash treated soil," Multiscale and Multidisciplinary Modeling, Experiments and Design, vol. 4, no. 8, 2021b.

[37] S. D. Mohammadzadeh, S.-F. Kazemi, A. Mosavi, E. Nasseralshariati, and J. H. M. Tah, "Prediction of compression index of fine-grained soils using a gene expression programming model," Infrastructures, vol. 4, pp. 1-12, 2019. 